\title{
Grain boundaries in the Swift-Hohenberg equation
}

\author{
Mariana Haragus ${ }^{a} \&$ Arnd Scheel $^{b}$ \\ ${ }^{a}$ Université de Franche-Comté, Laboratoire de Mathématiques, 25030 Besançon cedex, France \\ ${ }^{b}$ University of Minnesota, School of Mathematics, 206 Church St. S.E., Minneapolis, MN 55455, USA
}

July 11, 2012

\begin{abstract}
We study the existence of grain boundaries in the Swift-Hohenberg equation. The analysis relies on a spatial dynamics formulation of the existence problem and a centre-manifold reduction. In this setting, the grain boundaries are found as heteroclinic orbits of a reduced system of ODEs in normal form. We show persistence of the leading-order approximation using transversality induced by wavenumber selection.
\end{abstract}

Acknowledgments This work was partially supported by the Agence Nationale de la Recherche through grant ANR PREFERED (M.H.) and the National Science Foundation through grant NSF-DMS-0806614 (A.S.).

Running head: Grain boundaries in the Swift-Hohenberg equation

Corresponding author: Mariana Haragus, mharagus@univ-fcomte.fr

Keywords: Swift-Hohenberg equation, roll solution, grain boundary 


\section{Introduction}

When spatially periodic patterns emerge in isotropic pattern-forming systems, random initial conditions often lead to patches of patterns with different orientation that are separated by sharp interfaces. These interfaces play an important role in the selection of dominant wavenumbers, and their slow dynamics often govern the long-time behaviour of systems far from equilibrium. In an analogy to solid state physics, patches of uniformly oriented patterns correspond to crystallites and interfaces separating different orientations are then referred to as grain boundaries. To fix ideas, consider the Swift-Hohenberg equation

$$
\partial_{t} u=-(1+\Delta)^{2} u+\mu u-u^{3}
$$

in which $u$ depends upon two spatial variables $(x, y) \in \mathbb{R}^{2}$ and time $t \in \mathbb{R}$, and where $\mu$ is a real parameter. For $\mu>0$, small, one can readily show the existence of solutions $u_{\mathrm{r}}(k x ; k, \mu)$ which are spatially periodic $u_{\mathrm{r}}(\xi ; k, \mu)=u_{\mathrm{r}}(\xi+2 \pi ; k, \mu)$, and even in $\xi$. Interpreting Swift-Hohenberg as a toy model for instabilities in fluid systems such as Rayleigh-Bénard convection, in which such spatially periodic patterns correspond to convection rolls, we refer to such patterns as roll solutions; see for instance [1]. Roll solutions exist in the Swift-Hohenberg equation for $\mu>\mu_{*}(k)=\left(1-k^{2}\right)^{2}, \mu \sim$ $0, k \sim 1$. Rotational isotropy guarantees the existence of a family of rotated roll solutions

$$
u_{\mathrm{r}}^{\varphi}(x, y ; k):=u_{\mathrm{r}}(k(x \cos \varphi-y \sin \varphi) ; k, \mu),
$$

with $\varphi \in[0,2 \pi)$. Roll solutions are known to be stable for a range of wavenumbers and parameter values, $\sqrt{\mu / 12}+\mathrm{O}(\mu)>k-1>-\mu^{2} / 1024+\mathrm{O}\left(\mu^{3 / 2}\right) ;[1,14,17]$.

Near onset, Swift-Hohenberg and other pattern-forming systems are well approximated by modulation equations that describe the evolution of the complex amplitude $A$ of linear modes $\mathrm{e}^{\mathrm{i} x}$. At leading order one finds the Newell-Whitehead-Segel equations [17, 21],

$$
A_{T}=\left(\partial_{x}-\mathrm{i} \partial_{y y}\right)^{2} A+A-A|A|^{2} .
$$

Of course, the evolution of rotated modes, $\mathrm{e}^{\mathrm{i}(x \cos \varphi+y \sin \varphi)}$ is governed by the same equation. Since the initial conditions may lead to the presence of more than one, possibly a continuum of wavenumbers, the evolution of systems like the Swift-Hohenberg equation near onset may well require several coupled amplitude equations of the form (1.3), possibly even a continuum in some cases. Grain boundaries have been studied within such a leading-order approximation [13]. Our approach starts with the SwiftHohenberg equation and finds coupled amplitude equations as leading-order terms in a particular reduced system, allowing us to treat higher-order perturbations in a rigorous and systematic fashion.

We construct the grain boundaries as solutions to the equation (1.1) which are steady and periodic in $y$ with wavenumber $k$. Rescaling $y$, we find grain boundaries as solutions to

$$
0=-\left(1+\partial_{x}^{2}+k^{2} \partial_{y}^{2}\right)^{2} u+\mu u-u^{3}, \quad \partial_{y}^{j} u(x, 0)=\partial_{y}^{j} u(x, 2 \pi), j=0,1,2,3 .
$$

Grain boundaries are particular solutions $u_{\mathrm{gb}}(x, y)$ to $(1.4)$ that converge to rotated roll solutions,

$$
\left|u_{\mathrm{gb}}\left(x-x_{ \pm}, y\right)-u_{\mathrm{r}}^{\varphi_{ \pm}}\left(x, y ; k_{ \pm}\right)\right| \rightarrow 0 \text { for } x \rightarrow \pm \infty .
$$

Here, the constants are suitable asymptotic phases $x_{ \pm}$, asymptotic angles $\varphi_{ \pm}$, and asymptotic wavenumbers $k_{ \pm}$. Convergence is in fact exponential, and uniform in $y$, also for $y$-derivatives. The asymptotic 

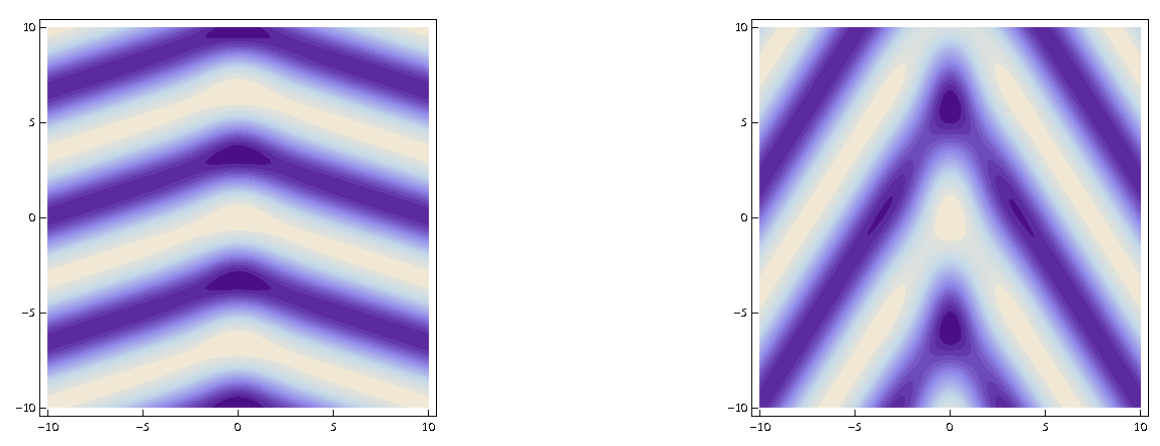

Figure 1.1: Plot of leading-order approximation to grain boundaries using the numerically computed heteroclinic solution to (4.2) and the leading-order coordinates on the centre manifold. The left picture shows $\varphi_{-}-\varphi_{+}=4 \pi / 5$, the right picture shows $\varphi_{-}-\varphi_{+}=4 \pi / 11$.

roll solutions $u_{\mathrm{r}}^{\varphi}$ were defined in (1.2). We also refer to Figure 2.1, below, for a schematic illustration of the various angles and wavenumbers related to a grain boundary.

We study existence of such grain boundaries for $\mu$ small under two main assumptions:

- symmetry: $u_{\mathrm{gb}}(x, y)=u_{\mathrm{gb}}(-x, y)$; in particular, $\varphi_{+}=-\varphi_{-}$.

- non-small angles: the angle between asymptotic rolls is not small, $\varphi_{-}-\varphi_{+}>\pi / 3$.

We emphasize that both $\varphi_{+}$and $\varphi_{-}$are well defined as angles of the rolls relative to the grain boundary interface, since periodicity in $y$ fixes the orientation of the interface, hence effectively factoring the rotational symmetry. Symmetry also fixes the location $x$. Using translation in $y$, we can assume that $x_{ \pm}=0$.

Our main result states existence of such grain boundaries for small values of $\mu$.

Theorem 1 For every angle $\varphi_{-}-\varphi_{+}=\alpha \in(\pi / 3, \pi)$ and $\mu$ sufficiently small, there exists a symmetric grain boundary with nearby angle $\alpha+\mathrm{O}(\mu)$ and asymptotic wavenumber $k_{ \pm}=1+\mathrm{O}(\mu)$.

We computed the leading-order approximation on the centre manifold numerically using AUTO07P and plotted the resulting leading-order approximations for grain boundaries in Figure 1.1.

Reducing formally to amplitude equations, grain boundaries have been studied in [13]. Subtle transitions in the structure of grain boundaries have been analyzed within the phase-diffusion equation in $[3,5]$. An existence proof for grain boundaries with angle $\alpha=\pi-\varepsilon$ was given in [8]. Rather than expanding near onset, $\mu \sim 0$, the analysis there is based on an expansion near a zigzag instability, for fixed $\mu>0$, small. The analysis there generalizes easily to roll patterns near a zigzag instability far from onset since only phase information, that is, response to local translations of the pattern, is used. The leading-order description resembles a Cahn-Hilliard equation [2] and grain boundaries correspond to explicit kink solutions in the Cahn-Hilliard equation. Since at leading order only the position and orientation of rolls is modulated in these grain boundaries, they are commonly referred to as knee solutions, emphasizing the bending aspect rather than the defect nature.

Our proof is based on a spatial-dynamics formulation, a centre-manifold reduction, normal form transformations, and a perturbation analysis of a leading-order coupled mode reduced system. These tools 
have been extensively used to solve concrete problems arising in physics and natural sciences (e.g., see $[7,11,15,16,20]$ and the references therein). Spatial dynamics goes back to the work of Kirchgässner [12]. It consists of studying a stationary problem by writing it as an evolutionary system in which an unbounded spatial coordinate plays the role of the time-like variable. The resulting evolutionary problem is typically ill-posed, but its small bounded solutions can be found by a centre-manifold reduction, which shows that this system is locally equivalent to a finite-dimensional reduced dynamical system (e.g., see [7, 10, 15, 24]). Normal form theory provides an efficient way of studying this reduced system, by transforming it to a simpler system containing a minimal number of nonlinear terms (e.g., see $[7,10,25])$. We emphasize that we do not rely on variational structure or the reflection symmetry $u \mapsto-u$ : we exploit variational structure only at leading order, an artifact of the leading-order expansion, and we use the fact that quadratic terms of a centre-manifold expansion vanish. We comment on generalizations to other pattern-forming systems in the discussion.

Remark 1.1 Theorem 1 shows that grain boundaries select $k_{ \pm}$, the asymptotic wavenumbers. In fact, $k_{ \pm}=1$ to leading order for all values of the angle $\varphi_{ \pm}$. By this, we understand that grain boundaries exist for arbitrary angle $\varphi_{ \pm}$, but specific wavenumbers $k_{ \pm}$. The proof of the theorem shows that these grain boundaries are unique for a given angle in a certain sense. More precisely, there do not exist solutions that are close to the solutions we find in Theorem 1 in a local topology, but that converge to rolls with different wavenumbers.

Wavenumber selection by grain boundaries has been observed experimentally, in numerical simulations, and in amplitude equation approximations (see [13] and references therein). Our analysis shows that such a selection is valid beyond amplitude equation, a non-trivial aspect since the averaging procedure in amplitude equations often introduces artificial symmetries.

Phenomenologically, one observes that at interfaces between patches of rolls with different orientations, the wavenumber of the rolls adjusts to a selected wavenumber, first near the interface and then further away in the far field. Establishing such dynamic selection mechanisms rigorously appears to be difficult.

Outline: We formulate the problem of existence as a heteroclinic-connection problem in a spatial dynamics formulation and reduce the infinite-dimensional problem to a finite-dimensional ODE in Section 2. Section 3 contains a sequence of normal form transformations that distill a coupled mode problem at leading order. Section 4 shows existence and persistence of heteroclinic orbits and concludes with the proof of our main theorem. We conclude with a short discussion.

\section{Spatial dynamics and reduction}

Dynamical system. We rewrite the equation for grain boundaries (1.4) as a first order system,

$$
\frac{d U}{d x}=\mathcal{A}(\mu, k) U+\mathcal{F}(U)
$$

in which

$$
U=\left(\begin{array}{c}
u \\
u_{1} \\
v \\
v_{1}
\end{array}\right), \quad \mathcal{A}(\mu, k)=\left(\begin{array}{cccc}
0 & 1 & 0 & 0 \\
-\left(1+k^{2} \partial_{y}^{2}\right) & 0 & 1 & 0 \\
0 & 0 & 0 & 1 \\
\mu & 0 & -\left(1+k^{2} \partial_{y}^{2}\right) & 0
\end{array}\right), \quad \mathcal{F}(U)=\left(\begin{array}{c}
0 \\
0 \\
0 \\
-u^{3}
\end{array}\right)
$$



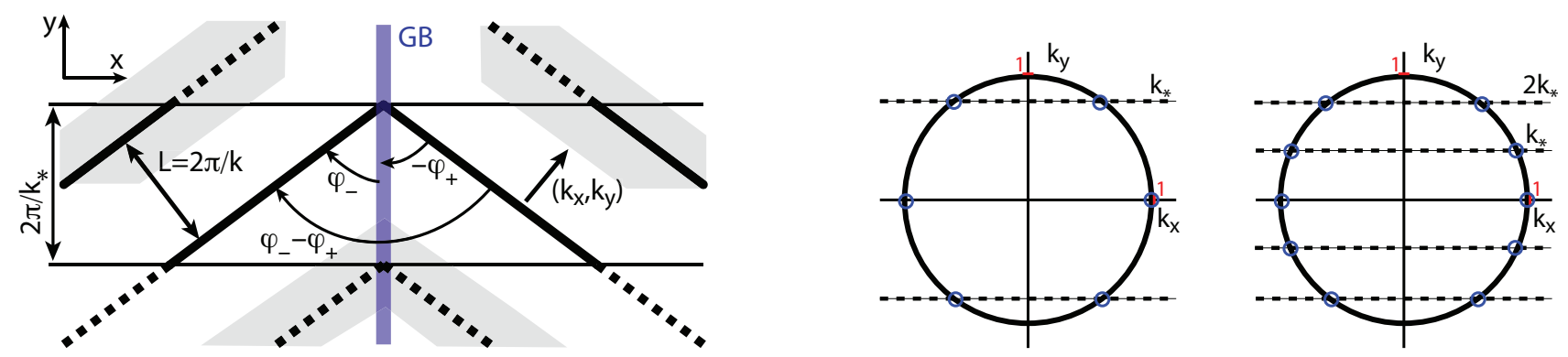

Figure 2.1: Orientation of rolls and wave vector relative to grain boundary interface and imposed boundary conditions on the left. On the right, roll orientations compatible with the periodicity in $y$ viewed in wavenumber space, with $k_{*}>1 / 2$ on the left and $k_{*}<1 / 2$ on the right. Small circles on the unit circle show critical wavenumbers that are compatible with the imposed periodicity in $y$.

We regard (2.1) as a dynamical system in the infinite-dimensional phase space

$$
\mathcal{X}=H_{\text {per }}^{3}(0,2 \pi) \times H_{\text {per }}^{2}(0,2 \pi) \times H_{\text {per }}^{1}(0,2 \pi) \times L^{2}(0,2 \pi),
$$

consisting of $2 \pi$-periodic functions, where

$$
H_{\mathrm{per}}^{j}(0,2 \pi)=\left\{u \in H_{\mathrm{loc}}^{j}(\mathbb{R}) ; u(z+2 \pi)=u(z), \forall z \in \mathbb{R}\right\}, \quad j \geqslant 1 .
$$

The linear part $\mathcal{A}(\mu, k)$ is a closed linear operator with dense domain

$$
\mathcal{Y}=H_{\mathrm{per}}^{4}(0,2 \pi) \times H_{\mathrm{per}}^{3}(0,2 \pi) \times H_{\mathrm{per}}^{2}(0,2 \pi) \times H_{\mathrm{per}}^{1}(0,2 \pi),
$$

and the nonlinear map $\mathcal{F}: \mathcal{Y} \rightarrow \mathcal{Y}$ is smooth.

Choice of parameters. We fix the wavenumber $k=k_{*}$ such that

$$
\frac{1}{2}<k_{*}<1
$$

and take $\mu$ as small bifurcation parameter. Since bifurcating rolls have wavenumber $\left(k_{x}, k_{y}\right)=$ $\left(k_{0} \cos \varphi,-k_{0} \sin \varphi\right)$ with $k_{0} \sim 1$, the above choice of $k_{*}=\left|k_{y}\right|$ corresponds to angles $\pi / 6<\varphi_{-}=$ $-\varphi_{+}<\pi / 2$, implying a non-small angle $\alpha=\varphi_{-}-\varphi_{+}>\pi / 3$ between grain boundaries. We note here that this condition guarantees that only rolls with angles $\varphi= \pm \arcsin \left(k_{*}\right)$ and $\varphi=0$ are compatible with the periodic boundary conditions in $y$; see Figure 2.1. For $k_{*} \leqslant 1 / 2$, orientations with $\varphi= \pm \arcsin \left(2 k_{*}\right)$ are compatible as well, conceivably leading to resonances as will become apparent in the linear analysis below; see also the discussion.

We set

$$
\mathcal{A}_{*}=\mathcal{A}\left(0, k_{*}\right), \quad \mathcal{B}(\mu)=\mathcal{A}\left(\mu, k_{*}\right)-\mathcal{A}\left(0, k_{*}\right)
$$

and write the system (2.1) in the form

$$
\frac{d U}{d x}=\mathcal{A}_{*} U+\mathcal{B}(\mu) U+\mathcal{F}(U)
$$


Central space. The linear operator $\mathcal{A}_{*}$ is closed in $\mathcal{X}$, with dense and compactly embedded domain $\mathcal{Y}$. Then $\mathcal{A}_{*}$ has compact resolvent, and its spectrum $\sigma\left(\mathcal{A}_{*}\right)$ is a purely point spectrum, only. The eigenvalues $\nu$ of $\mathcal{A}_{*}$ are determined by the dispersion relation,

$$
\nu^{2}=k_{*}^{2} \ell^{2}-1, \quad \ell \in \mathbb{Z},
$$

so that

$$
\sigma\left(\mathcal{A}_{*}\right)=\left\{\nu \in \mathbb{C} ; \nu^{2}=k_{*}^{2} \ell^{2}-1, \ell \in \mathbb{Z}\right\} .
$$

Taking into account the restriction on non-small angles (2.2) this implies that

$$
\sigma\left(\mathcal{A}_{*}\right) \cap \mathrm{i} \mathbb{R}=\left\{ \pm \mathrm{i}, \pm \mathrm{i} k_{x}\right\}, \quad k_{x}=\sqrt{1-k_{*}^{2}}
$$

see also Figure 2.1.

The eigenvalues $\pm \mathrm{i}$ are geometrically simple and algebraically double. The generalized eigenspace associated with the eigenvalue $\mathrm{i}$ is spanned by

$$
E_{0}(y)=\left(\begin{array}{l}
1 \\
\mathrm{i} \\
0 \\
0
\end{array}\right), \quad F_{0}(y)=\left(\begin{array}{c}
0 \\
1 \\
2 \mathrm{i} \\
-2
\end{array}\right)
$$

which satisfy

$$
\mathcal{A}_{*} E_{0}=\mathrm{i} E_{0}, \quad \mathcal{A}_{*} F_{0}=\mathrm{i} F_{0}+E_{0},
$$

and the generalized eigenspace associated with the eigenvalue $-\mathrm{i}$ is spanned by the complex conjugated vectors $\overline{E_{0}}$ and $\overline{F_{0}}$. The eigenvalues $\pm \mathrm{i} k_{x}$ are geometrically double and algebraically quadruple. The generalized eigenspace associated with the eigenvalue $\mathrm{i} k_{x}$ is spanned by

$$
E_{ \pm}(y)=\left(\begin{array}{c}
1 \\
\mathrm{i} k_{x} \\
0 \\
0
\end{array}\right) \mathrm{e}^{ \pm \mathrm{i} y}, \quad F_{ \pm}(y)=\left(\begin{array}{c}
0 \\
1 \\
2 \mathrm{i} k_{x} \\
-2 k_{x}^{2}
\end{array}\right) \mathrm{e}^{ \pm \mathrm{i} y}
$$

which satisfy

$$
\mathcal{A}_{*} E_{ \pm}=\mathrm{i} k_{x} E_{ \pm}, \quad \mathcal{A}_{*} F_{ \pm}=\mathrm{i} k_{x} F_{ \pm}+E_{ \pm},
$$

and the generalized eigenspace associated with the eigenvalue $-\mathrm{i} k_{x}$ is spanned by the complex conjugated vectors $\overline{E_{ \pm}}$and $\overline{F_{ \pm}}$. We conclude that the central space $\mathcal{X}_{c}$ of the operator $\mathcal{A}_{*}$, which is the spectral subspace associated with the purely imaginary eigenvalues of $\mathcal{A}_{*}$, is twelve-dimensional and spanned by the vectors $\left\{E_{0}, F_{0}, E_{ \pm}, F_{ \pm}\right\}$and the complex conjugated vectors $\left\{\overline{E_{0}}, \overline{F_{0}}, \overline{E_{ \pm}}, \overline{F_{ \pm}}\right\}$.

We also need to compute the spectral projection $\mathcal{P}_{c}: \mathcal{X} \rightarrow \mathcal{X}_{c}$ onto this spectral subspace. Denoting by $\langle\cdot, \cdot\rangle$ the scalar product in $\left(L^{2}(0,2 \pi)\right)^{4}$, the spectral projection is given by

$$
\mathcal{P}_{c} U=\sum_{\kappa \in\{0, \pm\}}\left(\left\langle U, E_{\kappa}^{\mathrm{ad}}\right\rangle E_{\kappa}+\left\langle U, F_{\kappa}^{\mathrm{ad}}\right\rangle F_{\kappa}+\left\langle U, \overline{E_{\kappa}^{\mathrm{ad}}}\right\rangle \overline{E_{\kappa}}+\left\langle U, \overline{F_{\kappa}^{\mathrm{ad}}}\right\rangle \overline{F_{\kappa}}\right),
$$

where

$$
\left\langle E_{\kappa}, E_{\ell}^{\mathrm{ad}}\right\rangle=\left\langle F_{\kappa}, F_{\ell}^{\mathrm{ad}}\right\rangle=\delta_{\kappa \ell}, \quad\left\langle F_{\kappa}, E_{\ell}^{\mathrm{ad}}\right\rangle=\left\langle E_{\kappa}, F_{\ell}^{\mathrm{ad}}\right\rangle=0, \quad \kappa, \ell \in\{0, \pm\},
$$




$$
\mathcal{A}_{*}^{\mathrm{ad}} F_{0}^{\mathrm{ad}}=-\mathrm{i} F_{0}^{\mathrm{ad}}, \quad \mathcal{A}_{*}^{\mathrm{ad}} E_{0}^{\mathrm{ad}}=-\mathrm{i} E_{0}^{\mathrm{ad}}+F_{0}^{\mathrm{ad}}, \quad \mathcal{A}_{*}^{\mathrm{ad}} F_{ \pm}^{\mathrm{ad}}=-\mathrm{i} k_{x} F_{ \pm}^{\mathrm{ad}}, \quad \mathcal{A}_{*}^{\mathrm{ad}} E_{ \pm}^{\mathrm{ad}}=-\mathrm{i} k_{x} E_{ \pm}^{\mathrm{ad}}+F_{ \pm}^{\mathrm{ad}},
$$

and $\mathcal{A}_{*}^{\text {ad }}$ is the adjoint of $\mathcal{A}_{*}$ with respect to the scalar product $\langle\cdot, \cdot\rangle$,

$$
\mathcal{A}_{*}^{\mathrm{ad}}=\left(\begin{array}{cccc}
0 & -\left(1+k_{*}^{2} \partial_{y}^{2}\right) & 0 & 0 \\
1 & 0 & 0 & 0 \\
0 & 1 & 0 & -\left(1+k_{*}^{2} \partial_{y}^{2}\right) \\
0 & 0 & 1 & 0
\end{array}\right) .
$$

A direct calculation gives

$$
\begin{gathered}
E_{0}^{\mathrm{ad}}(y)=\frac{1}{8 \pi}\left(\begin{array}{c}
2 \\
2 \mathrm{i} \\
0 \\
\mathrm{i}
\end{array}\right), \quad F_{0}^{\mathrm{ad}}(y)=\frac{1}{8 \pi}\left(\begin{array}{c}
0 \\
0 \\
\mathrm{i} \\
-1
\end{array}\right) \\
E_{ \pm}^{\mathrm{ad}}(y)=\frac{1}{8 \pi k_{x}^{3}}\left(\begin{array}{c}
2 k_{x}^{3} \\
2 \mathrm{i} k_{x}^{2} \\
0 \\
\mathrm{i}
\end{array}\right) e^{ \pm \mathrm{i} y}, \quad F_{ \pm}^{\mathrm{ad}}(y)=\frac{1}{8 \pi k_{x}^{3}}\left(\begin{array}{c}
0 \\
0 \\
\mathrm{i} k_{x}^{2} \\
-k_{x}
\end{array}\right) e^{ \pm \mathrm{i} y} .
\end{gathered}
$$

Reduction to a centre manifold. In the next step, we perform a nonlinear reduction to a centre manifold, capturing all small bounded solutions to (1.4) that are $2 \pi$-periodic in $y$, for small parameters $\mu$.

We want to apply the centre-manifold theorem to the system (2.3). By construction, the spectrum of the restriction of $\mathcal{A}_{*}$ to the space $\left(\right.$ id $\left.-\mathcal{P}_{c}\right) \mathcal{X}$ satisfies

$$
\sigma\left(\left.\mathcal{A}_{*}\right|_{\left(\mathrm{id}-\mathcal{P}_{c}\right) \mathcal{X}}\right) \subset\{\lambda \in \mathbb{C} ;|\operatorname{Re} \lambda| \geqslant \delta\}
$$

for some $\delta>0$. A direct calculation shows that the resolvent operator $\left(\mathrm{i} \omega-\mathcal{A}_{*}\right)^{-1}$ satisfies

$$
\left\|\left(\mathrm{i} \omega-\mathcal{A}_{*}\right)^{-1}\right\|_{\mathcal{L}\left(\left(\mathrm{id}-\mathcal{P}_{c}\right) \mathcal{X}\right)} \leqslant \frac{C}{1+|\omega|}, \quad \forall \omega \in \mathbb{R},
$$

with some positive constant $C>0$. We can therefore apply the centre-manifold theorem (see for instance [7, Section 2.2]) and conclude that there exist three neighbourhoods of the origin $\mathcal{U} \subset \mathcal{X}_{c}$, $\mathcal{V} \subset\left(\right.$ id $\left.-\mathcal{P}_{c}\right) \mathcal{Y}, \mathcal{W} \subset \mathbb{R}$ and a map $\boldsymbol{\Psi}: \mathcal{U} \times \mathcal{W} \rightarrow \mathcal{V}$ of class $C^{m}$, for an arbitrary, but fixed $m \geqslant 1$, such that the following hold. First, for any $\mu \in \mathcal{W}$, the bounded solutions of $(2.3)$ with $U(x) \in \mathcal{U} \times \mathcal{V}$, for all $x \in \mathbb{R}$, lie on the centre manifold given by the graph of $\boldsymbol{\Psi}(\cdot, \mu)$, that is,

$$
U(x)=U_{c}(x)+\Psi\left(U_{c}(x), \mu\right), \quad \forall x \in \mathbb{R} .
$$

Moreover, the centre manifold is tangent to the centre eigenspace,

$$
\left\|\mathbf{\Psi}\left(U_{c}, \mu\right)\right\|_{\mathcal{Y}}=\mathcal{O}\left(|\mu|\left\|U_{c}\right\|+\left\|U_{c}\right\|^{2}\right) .
$$

Substituting (2.5) into (2.3) and projecting with $\mathcal{P}_{c}$, we obtain the reduced system for $U_{c}$,

$$
\frac{d U_{c}}{d x}=\mathcal{A}_{*} U_{c}+\mathcal{P}_{c}\left(\mathcal{B}(\mu)\left(U_{c}+\boldsymbol{\Psi}\left(U_{c}, \mu\right)\right)+\mathcal{F}\left(U_{c}+\Psi\left(U_{c}, \mu\right)\right)\right) .
$$


Reduced system. We compute the Taylor expansion up to order three of the vector field in the reduced system (2.7). First, notice that the estimate (2.6) and the fact that the nonlinearity $\mathcal{F}$ is cubic imply that

$$
\frac{d U_{c}}{d x}=\mathcal{A}_{*} U_{c}+\mathcal{P}_{c}\left(\mathcal{B}(\mu) U_{c}+\mathcal{F}\left(U_{c}\right)\right)+O\left(|\mu|^{2}\left\|U_{c}\right\|+|\mu|\left\|U_{c}\right\|^{2}+\left\|U_{c}\right\|^{4}\right) .
$$

Next, using the basis of $\mathcal{X}_{c}$ constructed above, we set

$$
U_{c}(x)=\sum_{\kappa \in\{0, \pm\}}\left(A_{\kappa}(x) E_{\kappa}+B_{\kappa}(x) F_{\kappa}+\overline{A_{\kappa}}(x) \overline{E_{\kappa}}+\overline{B_{\kappa}}(x) \overline{F_{\kappa}}\right)
$$

where $A_{\kappa}, B_{\kappa}, \kappa \in\{0, \pm\}$ are complex-valued functions. A straightforward but lengthy calculation gives the leading-order terms in the reduced system

$$
\frac{d U_{c}}{d x}=\mathcal{A}_{*} U_{c}+\mathcal{P}_{c}\left(\mathcal{B}(\mu) U_{c}+\mathcal{F}\left(U_{c}\right)\right)
$$

expressed in the basis $\left(A_{\kappa}, B_{\kappa}\right), \kappa \in\{0, \pm\}$, of the centre eigenspace,

$$
\begin{aligned}
A_{0}^{\prime} & =\mathrm{i} A_{0}+B_{0}-\frac{\mathrm{i}}{4}\left(\mu a_{0}-a_{0}\left(a_{0}^{2}+6 a_{+} \overline{a_{+}}\right)\right) \\
B_{0}^{\prime} & =\mathrm{i} B_{0}-\frac{1}{4}\left(\mu a_{0}-a_{0}\left(a_{0}^{2}+6 a_{+} \overline{a_{+}}\right)\right) \\
A_{+}^{\prime} & =\mathrm{i} k_{x} A_{+}+B_{+}-\frac{\mathrm{i}}{4 k_{x}^{3}}\left(\mu a_{+}-3 a_{+}\left(a_{0}^{2}+a_{+} \overline{a_{+}}\right)\right) \\
B_{+}^{\prime} & =\mathrm{i} k_{x} B_{+}-\frac{1}{4 k_{x}^{2}}\left(\mu a_{+}-3 a_{+}\left(a_{0}^{2}+a_{+} \overline{a_{+}}\right)\right) \\
A_{-}^{\prime} & =\mathrm{i} k_{x} A_{-}+B B_{-}-\frac{\mathrm{i}}{4 k_{x}^{3}}\left(\mu \overline{a_{+}}-3 \overline{a_{+}}\left(a_{0}^{2}+a_{+} \overline{a_{+}}\right)\right) \\
B_{-}^{\prime} & =\mathrm{i} k_{x} B_{-}-\frac{1}{4 k_{x}^{2}}\left(\mu \overline{a_{+}}-3 \overline{a_{+}}\left(a_{0}^{2}+a_{+} \overline{a_{+}}\right)\right)
\end{aligned}
$$

in which

$$
a_{0}=A_{0}+\overline{A_{0}}, \quad b_{0}=B_{0}+\overline{B_{0}}, \quad a_{+}=A_{+}+\overline{A_{-}}, \quad a_{-}=A_{+}-\overline{A_{-}}, \quad b_{+}=B_{+}+\overline{B_{-}} .
$$

Symmetries The Swift-Hohenberg equation (1.4) possesses three reflection symmetries

$$
y \mapsto-y, \quad x \mapsto-x, \quad u \mapsto-u .
$$

These symmetries are inherited by the dynamical system (2.1), and are preserved through the centremanifold reduction. As a consequence, the reduced system (2.7) inherits the induced symmetries. The reflection $y \mapsto-y$ implies that the vector field in the reduced system (2.7) commutes with

$$
\mathcal{S}_{1}\left(A_{0}, B_{0}, A_{+}, B_{+}, A_{-}, B_{-}\right)=\left(A_{0}, B_{0}, A_{-}, B_{-}, A_{+}, B_{+}\right),
$$

and the reflection $x \mapsto-x$ implies that the vector field is reversible, i.e., it anticommutes with

$$
\mathcal{R}\left(A_{0}, B_{0}, A_{+}, B_{+}, A_{-}, B_{-}\right)=\left(\overline{A_{0}},-\overline{B_{0}}, \overline{A_{-}},-\overline{B_{-}}, \overline{A_{+}},-\overline{B_{+}}\right) .
$$

The reflection $u \mapsto-u$ implies that the vector field in the reduced system (2.7) is odd in $U_{c}$, i.e., it commutes with

$$
\mathcal{S}_{2}\left(A_{0}, B_{0}, A_{+}, B_{+}, A_{-}, B_{-}\right)=-\left(A_{0}, B_{0}, A_{+}, B_{+}, A_{-}, B_{-}\right)
$$


As a consequence, the higher-order terms in the reduced system (2.8) are such that

$$
\frac{d U_{c}}{d x}=\mathcal{A}_{*} U_{c}+\mathcal{P}_{c}\left(\mathcal{B}(\mu) U_{c}+\mathcal{F}\left(U_{c}\right)\right)+O\left(|\mu|^{2}\left\|U_{c}\right\|+|\mu|\left\|U_{c}\right\|^{3}+\left\|U_{c}\right\|^{5}\right) .
$$

Furthermore, as a consequence of the invariance of the Swift-Hohenberg equation (1.4) under translations in $y$, this reduced system is equivariant under the action of the circle group

$$
\mathcal{T}_{\phi}\left(A_{0}, B_{0}, A_{+}, B_{+}, A_{-}, B_{-}\right)=\left(A_{0}, B_{0}, \mathrm{e}^{i \phi} A_{+}, \mathrm{e}^{i \phi} B_{+}, \mathrm{e}^{-i \phi} A_{-}, \mathrm{e}^{-i \phi} B_{-}\right), \quad \phi \in \mathbb{R} / 2 \pi \mathbb{Z} .
$$

Remark 2.1 At this point, we exploited the symmetry $u \mapsto-u$, which is not present in general patternforming systems. It is not difficult to see, exploiting the normal form analysis in the following section, that absence of quadratic terms is sufficient, that is, we only require this symmetry to leading order. We comment on the effect of symmetry-breaking terms in Section 5.

\section{Normal form transformations}

In this section, we simplify (2.10) using a sequence of linear and nonlinear transformations. In a first step, we simplify the cubic terms in (2.9). We then simplify the parameter dependence of the linear part to arrive at a normal form that will be convenient for our study.

Cubic transformation. We consider the leading order of system (2.9) with $\mu=0$,

$$
\begin{aligned}
& A_{0}^{\prime}=\mathrm{i} A_{0}+B_{0}+\frac{\mathrm{i}}{4} a_{0}\left(a_{0}^{2}+6 a_{+} \overline{a_{+}}\right) \\
& B_{0}^{\prime}=\mathrm{i} B_{0}+\frac{1}{4} a_{0}\left(a_{0}^{2}+6 a_{+} \overline{a_{+}}\right) \\
& A_{+}^{\prime}=\mathrm{i} k_{x} A_{+}+B_{+}+\frac{3 \mathrm{i}}{4 k_{x}^{3}} a_{+}\left(a_{0}^{2}+a_{+} \overline{a_{+}}\right) \\
& B_{+}^{\prime}=\mathrm{i} k_{x} B_{+}+\frac{3}{4 k_{x}^{2}} a_{+}\left(a_{0}^{2}+a_{+} \overline{a_{+}}\right) \\
& A_{-}^{\prime}=\mathrm{i} k_{x} A_{-}+B_{-}+\frac{3 \mathrm{i}}{4 k_{x}^{3}} \overline{a_{+}}\left(a_{0}^{2}+a_{+} \overline{a_{+}}\right) \\
& B_{-}^{\prime}=\mathrm{i} k_{x} B_{-}+\frac{3}{4 k_{x}^{2}} \overline{a_{+}}\left(a_{0}^{2}+a_{+} \overline{a_{+}}\right) .
\end{aligned}
$$

Lemma 3.1 There exist homogeneous polynomials $\Phi_{0}, \Psi_{0}, \Phi_{ \pm}, \Psi_{ \pm}$of degree three in the complex variables $\mathbf{C}=\left(C_{0}, C_{ \pm}\right), \mathbf{D}=\left(D_{0}, D_{ \pm}\right)$, and their complex conjugates, such that the change of variables

$$
\begin{array}{cc}
A_{0}=C_{0}+\Phi_{0}(\mathbf{C}, \mathbf{D}, \overline{\mathbf{C}}, \overline{\mathbf{D}}), & B_{0}=D_{0}+\Psi_{0}(\mathbf{C}, \mathbf{D}, \overline{\mathbf{C}}, \overline{\mathbf{D}}) \\
A_{ \pm}=C_{ \pm}+\Phi_{ \pm}(\mathbf{C}, \mathbf{D}, \overline{\mathbf{C}}, \overline{\mathbf{D}}), & B_{ \pm}=D_{ \pm}+\Psi_{ \pm}(\mathbf{C}, \mathbf{D}, \overline{\mathbf{C}}, \overline{\mathbf{D}}),
\end{array}
$$


is well-defined in a neighbourhood of the origin and transforms the system (3.1) into the normal form

$$
\begin{aligned}
C_{0}^{\prime}= & \mathrm{i} C_{0}+D_{0}+O\left((|\mathbf{C}|+|\mathbf{D}|)^{5}\right) \\
D_{0}^{\prime}= & \mathrm{i} D_{0}+\frac{3}{4} C_{0}\left(\left|C_{0}\right|^{2}+2\left|C_{+}\right|^{2}+2\left|C_{-}\right|^{2}\right)+\frac{3 \mathrm{i}}{4} D_{0}\left(\left|C_{0}\right|^{2}+2\left|C_{+}\right|^{2}+2\left|C_{-}\right|^{2}\right) \\
& +\frac{3 \mathrm{i}}{4} C_{0}\left(C_{0} \overline{D_{0}}+\overline{C_{0}} D_{0}+2\left(C_{+} \overline{D_{+}}+\overline{C_{+}} D_{+}\right)+2\left(C_{-} \overline{D_{-}}+\overline{C_{-}} D_{-}\right)\right)+O\left((|\mathbf{C}|+|\mathbf{D}|)^{5}\right) \\
C_{+}^{\prime}= & \mathrm{i} k_{x} C_{+}+D_{+}+O\left((|\mathbf{C}|+|\mathbf{D}|)^{5}\right) \\
D_{+}^{\prime}= & \mathrm{i} k_{x} D_{+}+\frac{3}{4 k_{x}^{2}} C_{+}\left(2\left|C_{0}\right|^{2}+\left|C_{+}\right|^{2}+2\left|C_{-}\right|^{2}\right)+\frac{3 \mathrm{i}}{4 k_{x}^{3}} D_{+}\left(2\left|C_{0}\right|^{2}+\left|C_{+}\right|^{2}+2\left|C_{-}\right|^{2}\right) \\
& +\frac{3 \mathrm{i}}{4 k_{x}^{3}} C_{+}\left(2\left(C_{0} \overline{D_{0}}+\overline{C_{0}} D_{0}\right)+C_{+} \overline{D_{+}}+\overline{C_{+}} D_{+}+2\left(C_{-} \overline{D_{-}}+\overline{C_{-}} D_{-}\right)\right)+O\left((|\mathbf{C}|+|\mathbf{D}|)^{5}\right) \\
C_{-}^{\prime}= & \mathrm{i} k_{x} C_{-}+D_{-}+O\left((|\mathbf{C}|+|\mathbf{D}|)^{5}\right) \\
D_{-}^{\prime}= & \mathrm{i} k_{x} D_{-}+\frac{3}{4 k_{x}^{2}} C_{-}\left(2\left|C_{0}\right|^{2}+2\left|C_{+}\right|^{2}+\left|C_{-}\right|^{2}\right)+\frac{3 \mathrm{i}}{4 k_{x}^{3}} D_{-}\left(2\left|C_{0}\right|^{2}+2\left|C_{+}\right|^{2}+\left|C_{-}\right|^{2}\right) \\
& +\frac{3 \mathrm{i}}{4 k_{x}^{3}} C_{-}\left(2\left(C_{0} \overline{D_{0}}+\overline{C_{0}} D_{0}\right)+2\left(C_{+} \overline{D_{+}}+\overline{C_{+}} D_{+}\right)+C_{-} \overline{D_{-}}+\overline{C_{-}} D_{-}\right)+O\left((|\mathbf{C}|+|\mathbf{D}|)^{5}\right) .
\end{aligned}
$$

Proof. Denote by $\left(F_{0}, G_{0}, F_{+}, G_{+}, F_{-}, G_{-}\right)$and $\left(0, N_{0}, N_{+}, 0, N_{-}\right)$the cubic terms in the right hand sides of the systems (3.1) and (3.3), respectively. Following the proof of the normal form theorem (e.g., see [7, Section 3.1]), substituting (3.2) into (3.1), and taking into account (3.3) at order 3 in the resulting equalities we find that the polynomials $\Phi_{0}, \Psi_{0}, \Phi_{ \pm}, \Psi_{ \pm}$satisfy the equations

$$
\begin{array}{cl}
(\mathcal{D}-\mathrm{i}) \Phi_{0}=\Psi_{0}+F_{0}, & (\mathcal{D}-\mathrm{i}) \Psi_{0}=G_{0}-N_{0}, \\
\left(\mathcal{D}-\mathrm{i} k_{x}\right) \Phi_{ \pm}=\Psi_{ \pm}+F_{ \pm}, & \left(\mathcal{D}-\mathrm{i} k_{x}\right) \Psi_{ \pm}=G_{ \pm}-N_{ \pm},
\end{array}
$$

in which

$$
\begin{aligned}
\mathcal{D}= & \left(\mathrm{i} C_{0}+D_{0}\right) \frac{\partial}{\partial C_{0}}+\mathrm{i} D_{0} \frac{\partial}{\partial D_{0}}+\left(\mathrm{i} k_{x} C_{+}+D_{+}\right) \frac{\partial}{\partial C_{+}}+\mathrm{i} k_{x} D_{+} \frac{\partial}{\partial D_{+}} \\
& +\left(\mathrm{i} k_{x} C_{-}+D_{-}\right) \frac{\partial}{\partial C_{-}}+\mathrm{i} k_{x} D_{-} \frac{\partial}{\partial D_{-}}+\left(-\mathrm{i} \overline{C_{0}}+\overline{D_{0}}\right) \frac{\partial}{\partial \overline{C_{0}}}-\mathrm{i} \overline{D_{0}} \frac{\partial}{\partial \overline{D_{0}}} \\
& +\left(-\mathrm{i} k_{x} \overline{C_{+}}+\overline{D_{+}}\right) \frac{\partial}{\partial \overline{C_{+}}}-\mathrm{i} k_{x} \overline{D_{+}} \frac{\partial}{\partial \overline{D_{+}}}+\left(-\mathrm{i} k_{x} \overline{C_{-}}+\overline{D_{-}}\right) \frac{\partial}{\partial \overline{C_{-}}}-\mathrm{i} k_{x} \overline{D_{-}} \frac{\partial}{\partial \overline{D_{-}}} .
\end{aligned}
$$

The polynomials $\Phi_{0}, \Psi_{0}, \Phi_{ \pm}, \Psi_{ \pm}$exist provided the right hand sides in the equations (3.4) and (3.5) belong to the ranges of $\mathcal{D}-\mathrm{i}$ and $\mathcal{D}-\mathrm{i} k_{x}$, respectively.

We have

$$
F_{0}=\frac{\mathrm{i}}{4} P_{0}, \quad G_{0}=\frac{1}{4} P_{0}
$$

with

$$
P_{0}=C_{0}^{3}+3 C_{0}^{2} \overline{C_{0}}+3 C_{0}{\overline{C_{0}}}^{2}+{\overline{C_{0}}}^{3}+6\left(C_{0}+\overline{C_{0}}\right)\left(C_{+} \overline{C_{+}}+C_{+} C_{-}+\overline{C_{+} C_{-}}+C_{-} \overline{C_{-}}\right) .
$$

Notice that

$$
\begin{gathered}
(\mathcal{D}-\mathrm{i}) C_{0}^{3}=2 \mathrm{i} C_{0}^{3}+3 C_{0}^{2} D_{0}, \quad(\mathcal{D}-\mathrm{i}) C_{0}^{2} D_{0}=2 \mathrm{i} C_{0}^{2} D_{0}+2 C_{0} D_{0}^{2}, \\
(\mathcal{D}-\mathrm{i}) C_{0} D_{0}^{2}=2 \mathrm{i} C_{0} D_{0}^{2}+D_{0}^{3}, \quad(\mathcal{D}-\mathrm{i}) D_{0}^{3}=2 \mathrm{i} D_{0}^{3},
\end{gathered}
$$


which implies that $C_{0}^{3}$ belongs to the range of $\mathcal{D}$ - i. Similarly, we obtain that the monomials

$$
C_{0}{\overline{C_{0}}}^{2}, \quad{\overline{C_{0}}}^{3}, \quad C_{0} C_{+} C_{-}, \quad C_{0} \overline{C_{+} C_{-}}, \quad \overline{C_{0}} C_{+} \overline{C_{+}}, \quad \overline{C_{0}} C_{+} C_{-}, \quad \overline{C_{0} C_{+} C_{-}}, \quad \overline{C_{0}} C_{-} \overline{C_{-}}
$$

also belong to the range of $\mathcal{D}-\mathrm{i}$, whereas the monomials

$$
C_{0}^{2} \overline{C_{0}}, \quad C_{0} C_{+} \overline{C_{+}}, \quad C_{0} C_{-} \overline{C_{-}}
$$

do not belong to the range of $\mathcal{D}-\mathrm{i}$. Consequently, upon choosing

$$
\Psi_{0}=-\frac{3 \mathrm{i}}{4} C_{0}\left(\left|C_{0}\right|^{2}+2\left|C_{+}\right|^{2}+2\left|C_{-}\right|^{2}\right)+\widetilde{\Psi_{0}},
$$

with $\widetilde{\Psi_{0}}$ any element of the range of $\mathcal{D}-\mathrm{i}$, the polynomial $\Psi_{0}+F_{0}$ belongs to the range of $\mathcal{D}-\mathrm{i}$, so that there exists $\Phi_{0}$ satisfying the first equality in (3.4). Substituting (3.6) into the second equation in (3.4) we find

$$
\begin{aligned}
(\mathcal{D}-\mathrm{i}) \widetilde{\Psi_{0}}= & \frac{3 \mathrm{i}}{4} C_{0}\left(C_{0} \overline{D_{0}}+\overline{C_{0}} D_{0}+2\left(C_{+} \overline{D_{+}}+\overline{C_{+}} D_{+}\right)+2\left(C_{-} \overline{D_{-}}+\overline{C_{-}} D_{-}\right)\right) \\
& +\frac{3 \mathrm{i}}{4} D_{0}\left(\left|C_{0}\right|^{2}+2\left|C_{+}\right|^{2}+2\left|C_{-}\right|^{2}\right)+G_{0}-N_{0} .
\end{aligned}
$$

Taking

$$
\begin{aligned}
N_{0}= & \frac{3}{4} C_{0}\left(\left|C_{0}\right|^{2}+2\left|C_{+}\right|^{2}+2\left|C_{-}\right|^{2}\right)+\frac{3 \mathrm{i}}{4} D_{0}\left(\left|C_{0}\right|^{2}+2\left|C_{+}\right|^{2}+2\left|C_{-}\right|^{2}\right) \\
& +\frac{3 \mathrm{i}}{4} C_{0}\left(C_{0} \overline{D_{0}}+\overline{C_{0}} D_{0}+2\left(C_{+} \overline{D_{+}}+\overline{C_{+}} D_{+}\right)+2\left(C_{-} \overline{D_{-}}+\overline{C_{-}} D_{-}\right)\right)
\end{aligned}
$$

we find the equation

$$
(\mathcal{D}-\mathrm{i}) \widetilde{\Psi_{0}}=\frac{1}{4}\left(C_{0}^{3}+3 C_{0}{\overline{C_{0}}}^{2}+{\overline{C_{0}}}^{3}+6 C_{0}\left(C_{+} C_{-}+\overline{C_{+} C_{-}}\right)+\overline{C_{0}}\left(C_{+} \overline{C_{+}}+C_{+} C_{-}+\overline{C_{+} C_{-}}+C_{-} \overline{C_{-}}\right)\right),
$$

with right hand side belonging to the range of $\mathcal{D}-\mathrm{i}$. Consequently, $\widetilde{\Psi_{0}}$ exists, and it is not difficult to see that it also belongs to the range of $\mathcal{D}-$ i.

In a similar way, solving the equations (3.5) we obtain the polynomials

$$
\begin{aligned}
N_{+}= & \frac{3}{4 k_{x}^{2}} C_{+}\left(2\left|C_{0}\right|^{2}+\left|C_{+}\right|^{2}+2\left|C_{-}\right|^{2}\right)+\frac{3 \mathrm{i}}{4 k_{x}^{3}} D_{+}\left(2\left|C_{0}\right|^{2}+\left|C_{+}\right|^{2}+2\left|C_{-}\right|^{2}\right) \\
& +\frac{3 \mathrm{i}}{4 k_{x}^{3}} C_{+}\left(2\left(C_{0} \overline{D_{0}}+\overline{C_{0}} D_{0}\right)+C_{+} \overline{D_{+}}+\overline{C_{+}} D_{+}+2\left(C_{-} \overline{D_{-}}+\overline{C_{-}} D_{-}\right)\right) \\
N_{-}= & \frac{3}{4 k_{x}^{2}} C_{-}\left(2\left|C_{0}\right|^{2}+2\left|C_{+}\right|^{2}+\left|C_{-}\right|^{2}\right)+\frac{3 \mathrm{i}}{4 k_{x}^{3}} D_{-}\left(2\left|C_{0}\right|^{2}+2\left|C_{+}\right|^{2}+\left|C_{-}\right|^{2}\right) \\
& +\frac{3 \mathrm{i}}{4 k_{x}^{3}} C_{-}\left(2\left(C_{0} \overline{D_{0}}+\overline{C_{0}} D_{0}\right)+2\left(C_{+} \overline{D_{+}}+\overline{C_{+}} D_{+}\right)+C_{-} \overline{D_{-}}+\overline{C_{-}} D_{-}\right),
\end{aligned}
$$

and the existence of $\Phi_{ \pm}, \Psi_{ \pm}$, which proves the lemma. 
Parameter-dependent linear transformation. Consider the linear part of the system (2.9)

$$
\begin{aligned}
& \left.A_{0}^{\prime}=\mathrm{i} A_{0}+B_{0}-\frac{\mathrm{i}}{4}\left(\mu\left(A_{0}+\overline{A_{0}}\right)+B_{0}+\overline{B_{0}}\right)\right) \\
& \left.B_{0}^{\prime}=\mathrm{i} B_{0}-\frac{1}{4}\left(\mu\left(A_{0}+\overline{A_{0}}\right)+B_{0}+\overline{B_{0}}\right)\right) \\
& \left.A_{+}^{\prime}=\mathrm{i} k_{x} A_{+}+B_{+}-\frac{\mathrm{i}}{4 k_{x}^{3}}\left(\mu\left(A_{+}+\overline{A_{-}}\right)+B_{+}+\overline{B_{-}}\right)\right) \\
& \left.B_{+}^{\prime}=\mathrm{i} k_{x} B_{+}-\frac{1}{4 k_{x}^{2}}\left(\mu\left(A_{+}+\overline{A_{-}}\right)+B_{+}+\overline{B_{-}}\right)\right) \\
& \left.A_{-}^{\prime}=\mathrm{i} k_{x} A_{-}+B_{-}-\frac{\mathrm{i}}{4 k_{x}^{3}}\left(\mu\left(A_{-}+\overline{A_{+}}\right)+B_{-}+\overline{B_{+}}\right)\right) \\
& \left.B_{-}^{\prime}=\mathrm{i} k_{x} B_{-}-\frac{1}{4 k_{x}^{2}}\left(\mu\left(A_{-}+\overline{A_{+}}\right)+B_{-}+\overline{B_{+}}\right)\right) .
\end{aligned}
$$

Lemma 3.2 There exist linear maps $L_{0}, T_{0}, L_{ \pm}, T_{ \pm}$such that, for sufficiently small $\mu$, the linear change of variables

$$
\begin{array}{cl}
A_{0}=C_{0}+\mu L_{0}(\mathbf{C}, \mathbf{D}, \overline{\mathbf{C}}, \overline{\mathbf{D}}), & B_{0}=D_{0}+\mu T_{0}(\mathbf{C}, \mathbf{D}, \overline{\mathbf{C}}, \overline{\mathbf{D}}) \\
A_{ \pm}=C_{ \pm}+\mu L_{ \pm}(\mathbf{C}, \mathbf{D}, \overline{\mathbf{C}}, \overline{\mathbf{D}}), & B_{ \pm}=D_{ \pm}+\mu T_{ \pm}(\mathbf{C}, \mathbf{D}, \overline{\mathbf{C}}, \overline{\mathbf{D}})
\end{array}
$$

transforms the system (3.7) into the normal form

$$
\begin{aligned}
C_{0}^{\prime} & =\mathrm{i} C_{0}+D_{0}+O\left(|\mu|^{2}(|\mathbf{C}|+|\mathbf{D}|)\right) \\
D_{0}^{\prime} & =\mathrm{i} D_{0}-\frac{1}{4} \mu C_{0}-\frac{\mathrm{i}}{4} \mu D_{0}+O\left(|\mu|^{2}(|\mathbf{C}|+|\mathbf{D}|)\right) \\
C_{+}^{\prime} & =\mathrm{i} k_{x} C_{+}+D_{+}+O\left(|\mu|^{2}(|\mathbf{C}|+|\mathbf{D}|)\right) \\
D_{+}^{\prime} & =\mathrm{i} k_{x} D_{+}-\frac{1}{4 k_{x}^{2}} \mu C_{+}-\frac{\mathrm{i}}{4 k_{x}^{3}} \mu D_{+}+O\left(|\mu|^{2}(|\mathbf{C}|+|\mathbf{D}|)\right) \\
C_{-}^{\prime} & =\mathrm{i} k_{x} C_{-}+D_{-}+O\left(|\mu|^{2}(|\mathbf{C}|+|\mathbf{D}|)\right) \\
D_{-}^{\prime} & =\mathrm{i} k_{x} D_{-}-\frac{1}{4 k_{x}^{2}} \mu C_{-}-\frac{\mathrm{i}}{4 k_{x}^{3}} \mu D_{-}+O\left(|\mu|^{2}(|\mathbf{C}|+|\mathbf{D}|)\right)
\end{aligned}
$$

Proof. We proceed as in the proof of Lemma 3.1, now using the fact that $C_{0}$ (resp. $C_{ \pm}$) is the only variable which does not belong to the range of $\mathcal{D}-\mathrm{i}\left(\operatorname{resp} . \mathcal{D}-\mathrm{i} k_{x}\right)$.

Normal form of the reduced system. Applying now the change of variables

$$
\begin{array}{cl}
A_{0}=C_{0}+\left(\mu L_{0}+\Phi_{0}\right)(\mathbf{C}, \mathbf{D}, \overline{\mathbf{C}}, \overline{\mathbf{D}}), & B_{0}=D_{0}+\left(\mu T_{0}+\Psi_{0}\right)(\mathbf{C}, \mathbf{D}, \overline{\mathbf{C}}, \overline{\mathbf{D}}) \\
A_{ \pm}=C_{ \pm}+\left(\mu L_{ \pm}+\Phi_{ \pm}\right)(\mathbf{C}, \mathbf{D}, \overline{\mathbf{C}}, \overline{\mathbf{D}}), & B_{ \pm}=D_{ \pm}+\left(\mu T_{ \pm}+\Psi_{ \pm}\right)(\mathbf{C}, \mathbf{D}, \overline{\mathbf{C}}, \overline{\mathbf{D}})
\end{array}
$$


to the reduced system (2.10) we obtain to leading order the normal form

$$
\begin{aligned}
C_{0}^{\prime}= & \mathrm{i} C_{0}+D_{0} \\
D_{0}^{\prime}= & \mathrm{i} D_{0}-\frac{1}{4} C_{0}\left(\mu-3\left(\left|C_{0}\right|^{2}+2\left|C_{+}\right|^{2}+2\left|C_{-}\right|^{2}\right)\right) \\
& -\frac{\mathrm{i}}{4} D_{0}\left(\mu-3\left(\left|C_{0}\right|^{2}+2\left|C_{+}\right|^{2}+2\left|C_{-}\right|^{2}\right)\right) \\
& +\frac{3 \mathrm{i}}{4} C_{0}\left(C_{0} \overline{D_{0}}+\overline{C_{0}} D_{0}+2\left(C_{+} \overline{D_{+}}+\overline{C_{+}} D_{+}\right)+2\left(C_{-} \overline{D_{-}}+\overline{C_{-}} D_{-}\right)\right) \\
C_{+}^{\prime}= & \mathrm{i} k_{x} C_{+}+D_{+} \\
D_{+}^{\prime}= & \mathrm{i} k_{x} D_{+}-\frac{1}{4 k_{x}^{2}} C_{+}\left(\mu-3\left(2\left|C_{0}\right|^{2}+\left|C_{+}\right|^{2}+2\left|C_{-}\right|^{2}\right)\right) \\
& -\frac{\mathrm{i}}{4 k_{x}^{3}} D_{+}\left(\mu-3\left(2\left|C_{0}\right|^{2}+\left|C_{+}\right|^{2}+2\left|C_{-}\right|^{2}\right)\right) \\
& +\frac{3 \mathrm{i}}{4 k_{x}^{3}} C_{+}\left(2\left(C_{0} \overline{D_{0}}+\overline{C_{0}} D_{0}\right)+C_{+} \overline{D_{+}}+\overline{C_{+}} D_{+}+2\left(C_{-} \overline{D_{-}}+\overline{C_{-}} D_{-}\right)\right) \\
C_{-}^{\prime}= & \mathrm{i} k_{x} C_{-}+D_{-} \\
D_{-}^{\prime}= & \mathrm{i} k_{x} D_{-}-\frac{1}{4 k_{x}^{2}} C_{-}\left(\mu-3\left(2\left|C_{0}\right|^{2}+2\left|C_{+}\right|^{2}+\left|C_{-}\right|^{2}\right)\right) \\
& -\frac{\mathrm{i}}{4 k_{x}^{3}} D_{-}\left(\mu-3\left(2\left|C_{0}\right|^{2}+2\left|C_{+}\right|^{2}+\left|C_{-}\right|^{2}\right)\right) \\
& +\frac{3 \mathrm{i}}{4 k_{x}^{3}} C_{-}\left(2\left(C_{0} \overline{D_{0}}+\overline{C_{0}} D_{0}\right)+2\left(C_{+} \overline{D_{+}}+\overline{C_{+}} D_{+}\right)+C_{-} \overline{D_{-}}+\overline{C_{-}} D_{-}\right) .
\end{aligned}
$$

The higher-order terms in this normal form are of order $O\left(|\mu|^{2}(|\mathbf{C}|+|\mathbf{D}|)+(|\mathbf{C}|+|\mathbf{D}|)^{5}\right)$.

\section{Existence of heteroclinic orbits}

We look for solutions of the system (3.10) in the form

$$
C_{0}(x)=\mathrm{e}^{\mathrm{i} x} \widetilde{C_{0}}, \quad D_{0}(x)=\mathrm{e}^{\mathrm{i} x} \widetilde{D_{0}}, \quad C_{ \pm}(x)=\mathrm{e}^{\mathrm{i} k_{x} x} \widetilde{C_{ \pm}}, \quad D_{ \pm}(x)=\mathrm{e}^{\mathrm{i} k_{x} x} \widetilde{D_{ \pm}} .
$$

With the scaling

$$
\widehat{x}=|\mu|^{1 / 2} x, \quad \widetilde{C_{\kappa}}=|\mu|^{1 / 2} \widehat{C_{\kappa}}, \quad \widetilde{D_{\kappa}}=|\mu| \widehat{D_{\kappa}}, \quad \kappa \in\{0, \pm\},
$$

we obtain the new system

$$
\begin{aligned}
& C_{0}^{\prime}=D_{0}+O\left(|\mu|^{1 / 2}\right) \\
& D_{0}^{\prime}=-\frac{1}{4} C_{0}\left(\operatorname{sign}(\mu)-3\left(\left|C_{0}\right|^{2}+2\left|C_{+}\right|^{2}+2\left|C_{-}\right|^{2}\right)\right)+O\left(|\mu|^{1 / 2}\right) \\
& C_{+}^{\prime}=D_{+}+O\left(|\mu|^{1 / 2}\right) \\
& D_{+}^{\prime}=-\frac{1}{4 k_{x}^{2}} C_{+}\left(\operatorname{sign}(\mu)-3\left(2\left|C_{0}\right|^{2}+\left|C_{+}\right|^{2}+2\left|C_{-}\right|^{2}\right)\right)+O\left(|\mu|^{1 / 2}\right) \\
& C_{-}^{\prime}=D_{-}+O\left(|\mu|^{1 / 2}\right) \\
& D_{-}^{\prime}=-\frac{1}{4 k_{x}^{2}} C_{-}\left(\operatorname{sign}(\mu)-3\left(2\left|C_{0}\right|^{2}+2\left|C_{+}\right|^{2}+\left|C_{-}\right|^{2}\right)\right)+O\left(|\mu|^{1 / 2}\right),
\end{aligned}
$$


in which we have dropped the hats. Notice that the $O\left(|\mu|^{1 / 2}\right)$ terms in this system are depending upon $x$ through highly oscillating terms $\mathrm{e}^{ \pm 2 \mathrm{i} x /|\mu|^{1 / 2}}$ and $\mathrm{e}^{ \pm 2 \mathrm{i} k_{x} x /|\mu|^{1 / 2}}$. Taking $\mu>0$ in the system above, we find

$$
\begin{aligned}
& C_{0}^{\prime \prime}=-\frac{1}{4} C_{0}+\frac{3}{4} C_{0}\left(\left|C_{0}\right|^{2}+2\left|C_{+}\right|^{2}+2\left|C_{-}\right|^{2}\right)+O\left(\mu^{1 / 2}\right) \\
& C_{+}^{\prime \prime}=-\frac{1}{4 k_{x}^{2}} C_{+}+\frac{3}{4 k_{x}^{2}} C_{+}\left(2\left|C_{0}\right|^{2}+\left|C_{+}\right|^{2}+2\left|C_{-}\right|^{2}\right)+O\left(\mu^{1 / 2}\right) \\
& C_{-}^{\prime \prime}=-\frac{1}{4 k_{x}^{2}} C_{-}+\frac{3}{4 k_{x}^{2}} C_{-}\left(2\left|C_{0}\right|^{2}+2\left|C_{+}\right|^{2}+\left|C_{-}\right|^{2}\right)+O\left(\mu^{1 / 2}\right) .
\end{aligned}
$$

\section{$4.1 \quad$ Rotated rolls}

The Swift-Hohenberg equation (1.1) possesses roll solutions

$$
u_{\mu, \kappa}(x)=\sqrt{4\left(\mu-\kappa^{2}\right) / 3} \cos (\sqrt{1+\kappa} x)+\mathrm{O}\left(\left|\mu-\kappa^{2}\right|^{3 / 2}\right),
$$

for small $\mu \in\left(0, \mu_{0}\right]$ and $\kappa^{2}<\mu$ (e.g., see [14]). Recall that due to the rotation invariance of the equation, for any $\left(\ell_{x}, \ell_{y}\right)$ such that $\ell_{x}^{2}+\ell_{y}^{2}=1$, we find the rotated rolls $u_{\mu, \kappa}\left(\ell_{x} x+\ell_{y} y\right)$. Upon taking

$$
\kappa=\varepsilon \mu^{1 / 2}\left(2 k_{x}+\varepsilon \mu^{1 / 2}\right), \quad \ell_{x}=\frac{k_{x}+\varepsilon \mu^{1 / 2}}{\sqrt{1+\kappa}}, \quad \ell_{y}=\frac{k_{*}}{\sqrt{1+\kappa}},
$$

we obtain a family of rotated rolls

$$
u_{\mu, \varepsilon}(x, y)=\frac{1}{\sqrt{3}} \mu^{1 / 2}\left(1-4 k_{x}^{2} \varepsilon^{2}\right)^{1 / 2}\left(\mathrm{e}^{\mathrm{i}\left(k_{x}+\varepsilon \mu^{1 / 2}\right) x} \mathrm{e}^{\mathrm{i} k_{*} y}+\mathrm{e}^{-\mathrm{i}\left(k_{x}+\varepsilon \mu^{1 / 2}\right) x} \mathrm{e}^{-\mathrm{i} k_{*} y}\right)+O\left(\mu^{3 / 2}+\varepsilon^{3} \mu\right),
$$

for small parameters $\mu$ and $\varepsilon$.

In our set-up, steady solutions of (1.1) are found in the form

$$
\begin{aligned}
u(x, y)= & \mu^{1 / 2}\left(C_{0}\left(\mu^{1 / 2} x\right) \mathrm{e}^{\mathrm{i} x}+\overline{C_{0}}\left(\mu^{1 / 2} x\right) \mathrm{e}^{-\mathrm{i} x}+C_{+}\left(\mu^{1 / 2} x\right) \mathrm{e}^{\mathrm{i} k_{x} x} \mathrm{e}^{\mathrm{i} k_{*} y}+C_{-}\left(\mu^{1 / 2} x\right) \mathrm{e}^{\mathrm{i} k_{x} x} \mathrm{e}^{-\mathrm{i} k_{*} y}\right. \\
& \left.+\overline{C_{+}}\left(\mu^{1 / 2} x\right) \mathrm{e}^{-\mathrm{i} k_{x} x} \mathrm{e}^{-\mathrm{i} k_{*} y}+\overline{C_{-}}\left(\mu^{1 / 2} x\right) \mathrm{e}^{-\mathrm{i} k_{x} x} \mathrm{e}^{\mathrm{i} k_{*} y}\right)+O(\mu),
\end{aligned}
$$

in which $C_{0}, C_{+}$, and $C_{-}$are solutions of the system (4.2). As a consequence, the family of rolls in (4.4) gives a family of periodic orbits for (4.2),

$$
\mathbf{P}_{\mu, \varepsilon}(x)=\left(0, \frac{1}{\sqrt{3}}\left(1-4 k_{x}^{2} \varepsilon^{2}\right)^{1 / 2} \mathrm{e}^{\mathrm{i} \varepsilon x}, 0\right)+O\left(\mu^{1 / 2}\right) .
$$

Notice that these periodic orbits are not reversible. In particular, the reversibility symmetry $\mathcal{R}$ generates a second family of periodic orbits

$$
\mathbf{Q}_{\mu, \varepsilon}(x)=\left(\mathcal{R} \mathbf{P}_{\mu, \varepsilon}\right)(-x)=\left(0,0, \frac{1}{\sqrt{3}}\left(1-4 k_{x}^{2} \varepsilon^{2}\right)^{1 / 2} \mathrm{e}^{\mathrm{i} \varepsilon x}\right)+O\left(\mu^{1 / 2}\right),
$$

which corresponds to the reflected rolls $u_{\mu, \varepsilon}(-x, y)$. 


\subsection{Heteroclinic orbit of the leading-order system}

Consider the system (4.2) with $\mu=0$. Restricting to $C_{0}=0$ and real-valued solutions $C_{+}$and $C_{-}$we find the system

$$
\begin{aligned}
& C_{+}^{\prime \prime}=-\frac{1}{4 k_{x}^{2}} C_{+}+\frac{3}{4 k_{x}^{2}} C_{+}\left(C_{+}^{2}+2 C_{-}^{2}\right) \\
& C_{-}^{\prime \prime}=-\frac{1}{4 k_{x}^{2}} C_{-}+\frac{3}{4 k_{x}^{2}} C_{-}\left(2 C_{+}^{2}+C_{-}^{2}\right) .
\end{aligned}
$$

According to [22] this system possesses a heteroclinic orbit $\left(C_{+}^{*}, C_{-}^{*}\right)$ with the following properties:

(i) $\lim _{x \rightarrow-\infty}\left(C_{+}^{*}(x), C_{-}^{*}(x)\right)=(1 / \sqrt{3}, 0)$ and $\lim _{x \rightarrow \infty}\left(C_{+}^{*}(x), C_{-}^{*}(x)\right)=(0,1 / \sqrt{3})$;

(ii) $C_{+}^{*}(x) \geqslant 0$ and $C_{-}^{*}(x) \geqslant 0$, for all $x \in \mathbb{R}$;

(iii) $C_{+}^{*}(x)=C_{-}^{*}(-x)$, for all $x \in \mathbb{R}$;

(iv) $C_{+}^{*}(x)=C_{-}^{*}(x)$ if and only if $x=0$;

(v) $C_{+}^{* 2}(x)+C_{-}^{* 2}(x) \leqslant 1 / 3$ and $C_{+}^{*}(x)+C_{-}^{*}(x) \geqslant 1 / \sqrt{3}$, for all $x \in \mathbb{R}$;

(see also [9] for further properties). In particular, the heteroclinic orbit $\left(0, C_{+}^{*}, C_{-}^{*}\right)$ is reversible.

The heteroclinic orbit represents a solution at leading order to our reduced systems in normal form that converges to roll solutions with opposite angle — it is precisely the grain boundary we were looking for. Our main goal now is to show that the heteroclinic actually persists as a solution for the reduced equation (4.2), when including higher-order correction terms. In particular, there we want to show that there is a heteroclinic orbit for (4.2), for small $\mu$, which connects two periodic orbits $\mathbf{P}_{\mu, \varepsilon}$, as $x \rightarrow \infty$, and $\mathbf{Q}_{\mu, \varepsilon}$, as $x \rightarrow-\infty$. This then gives a solution to the full infinite-dimensional dynamical system (2.1) and hence a solution to the Swift-Hohenberg equation (1.4) which is $2 \pi$-periodic in $y$, hence proving Theorem 1.

A key role in our persistence proof is played by the linear operator found by linearizing the system (4.2), with $\mu=0$, at $\left(0, C_{+}^{*}, C_{-}^{*}\right)$, i.e., by the linear operator $\mathcal{L}_{*}$ acting on $C_{0}, C_{+}, C_{-}$through

$$
\mathcal{L}_{*}\left(\begin{array}{c}
C_{0} \\
C_{+} \\
C_{-}
\end{array}\right)=\left(\begin{array}{c}
C_{0}^{\prime \prime}+\frac{1}{4} C_{0}-\frac{3}{2}\left(C_{+}^{* 2}+C_{-}^{* 2}\right) C_{0} \\
C_{+}^{\prime \prime}+\frac{1}{4 k_{x}^{2}} C_{+}-\frac{3}{4 k_{x}^{2}}\left(2\left(C_{+}^{* 2}+C_{-}^{* 2}\right) C_{+}+C_{+}^{* 2} \overline{C_{+}}+2 C_{+}^{*} C_{-}^{*}\left(C_{-}+\overline{C_{-}}\right)\right) \\
C_{-}^{\prime \prime}+\frac{1}{4 k_{x}^{2}} C_{-}-\frac{3}{4 k_{x}^{2}}\left(2\left(C_{+}^{* 2}+C_{-}^{* 2}\right) C_{-}+C_{-}^{* 2} \overline{C_{-}}+2 C_{+}^{*} C_{-}^{*}\left(C_{+}+\overline{C_{+}}\right)\right)
\end{array}\right) .
$$

We consider the space of exponentially decaying functions

$$
\mathcal{X}_{\eta}=\left\{\left(C_{0}, C_{+}, C_{-}, \overline{C_{0}}, \overline{C_{+}}, \overline{C_{-}}\right) \in\left(L_{\eta}^{2}\right)^{6}\right\}, \quad L_{\eta}^{2}=\left\{f: \mathbb{R} \rightarrow \mathbb{C} ; \int_{\mathbb{R}} \mathrm{e}^{2 \eta|x|}|f(x)|^{2}<\infty\right\},
$$

for $\eta>0$, in which $\mathcal{L}_{*}$ is closed with dense domain

$$
\mathcal{Y}_{\eta}=\left\{\left(C_{0}, C_{+}, C_{-}, \overline{C_{0}}, \overline{C_{+}}, \overline{C_{-}}\right) \in\left(H_{\eta}^{2}\right)^{6}\right\}, \quad H_{\eta}^{2}=\left\{f: \mathbb{R} \rightarrow \mathbb{C} ; f, f^{\prime}, f^{\prime \prime} \in L_{\eta}^{2}\right\} .
$$

We are interested in the properties of the restriction of $\mathcal{L}_{*}$ to the space of reversible functions

$$
\mathcal{X}_{\eta}^{r}=\left\{\left(C_{0}, C_{+}, C_{-}, \overline{C_{0}}, \overline{C_{+}}, \overline{C_{-}}\right) \in \mathcal{X}_{\eta} ; C_{0}(x)=\overline{C_{0}}(-x), C_{+}(x)=\overline{C_{-}}(-x), x \in \mathbb{R}\right\}
$$


Lemma 4.1 Assume $\eta>0$ is sufficiently small. Then the operator $\mathcal{L}_{*}$ acting in $\mathcal{X}_{\eta}^{r}$ is Fredholm with index -1 . The kernel of $\mathcal{L}_{*}$ is trivial, and the one-dimensional kernel of its $L^{2}$-adjoint is spanned by $\left(0, \mathrm{i} C_{+}^{*},-\mathrm{i} C_{-}^{*}, 0,-\mathrm{i} C_{+}^{*}, \mathrm{i} C_{-}^{*}\right)$.

Proof. Decomposing into real and imaginary parts,

$$
C_{\kappa}=U_{\kappa}+\mathrm{i} V_{\kappa}, \quad \kappa \in\{0, \pm\}
$$

we write $\mathcal{L}_{*}$ as a real matrix operator

$$
\mathcal{M}_{*}=\left(\begin{array}{ccc}
\mathcal{M}_{0} & 0 & 0 \\
0 & \mathcal{M}_{r} & 0 \\
0 & 0 & \mathcal{M}_{i}
\end{array}\right)
$$

with

$$
\begin{aligned}
& \mathcal{M}_{0}\left(\begin{array}{c}
U_{0} \\
V_{0}
\end{array}\right)=\left(\begin{array}{c}
U_{0}^{\prime \prime}+\frac{1}{4} U_{0}-\frac{3}{2}\left(C_{+}^{* 2}+C_{-}^{* 2}\right) U_{0} \\
V_{0}^{\prime \prime}+\frac{1}{4} V_{0}-\frac{3}{2}\left(C_{+}^{* 2}+C_{-}^{* 2}\right) V_{0}
\end{array}\right), \\
& \mathcal{M}_{r}\left(\begin{array}{c}
U_{+} \\
U_{-}
\end{array}\right)=\left(\begin{array}{c}
U_{+}^{\prime \prime}+\frac{1}{4 k_{x}^{2}} U_{+}-\frac{3}{4 k_{x}^{2}}\left(\left(3 C_{+}^{* 2}+2 C_{-}^{* 2}\right) U_{+}+4 C_{+}^{*} C_{-}^{*} U_{-}\right) \\
U_{-}^{\prime \prime}+\frac{1}{4 k_{x}^{2}} U_{-}-\frac{3}{4 k_{x}^{2}}\left(\left(2 C_{+}^{* 2}+3 C_{-}^{* 2}\right) U_{-}+4 C_{+}^{*} C_{-}^{*} U_{+}\right)
\end{array}\right), \\
& \mathcal{M}_{i}\left(\begin{array}{c}
V_{+} \\
V_{-}
\end{array}\right)=\left(\begin{array}{c}
V_{+}^{\prime \prime}+\frac{1}{4 k_{x}^{2}} V_{+}-\frac{3}{4 k_{x}^{2}}\left(C_{+}^{* 2}+2 C_{-}^{* 2}\right) V_{+} \\
V_{-}^{\prime \prime}+\frac{1}{4 k_{x}^{2}} V_{-}-\frac{3}{4 k_{x}^{2}}\left(2 C_{+}^{* 2}+C_{-}^{* 2}\right) V_{-}
\end{array}\right),
\end{aligned}
$$

acting in, respectively,

$$
X_{\eta}^{0}=\left(L_{\eta}^{2}\right)^{2}, \quad X_{\eta}^{r}=\left\{\left(U_{+}, U_{-}\right) \in\left(L_{\eta}^{2}\right)^{2} ; U_{+}(x)=U_{-}(-x), x \in \mathbb{R}\right\}
$$

and

$$
X_{\eta}^{i}=\left\{\left(V_{+}, V_{-}\right) \in\left(L_{\eta}^{2}\right)^{2} ; V_{+}(x)=-V_{-}(-x), x \in \mathbb{R}\right\} .
$$

The linear operator $\mathcal{M}_{r}$ has been studied in detail in [9]. The results in [9, Section 4.2] show that $\mathcal{M}_{r}$ is a Fredholm operator with index 0 in $\left(L^{2}\right)^{2}$ and has a one-dimensional kernel spanned by $\left(C_{+}^{\prime}, C_{-}^{\prime}\right)$. Consequently, $\mathcal{M}_{r}$ is a Fredholm operator with index 0 in $X_{\eta}^{r}$, for sufficiently small $\eta>0$, with a trivial kernel, since $\left(C_{+}^{\prime}, C_{-}^{\prime}\right)$ does not belong to $X_{\eta}^{r}$. In particular, this implies that $\mathcal{M}_{r}$ is invertible in $X_{\eta}^{r}$.

Next, the linear operator $\mathcal{M}_{0}$ is diagonal, and it is enough to study the scalar operator

$$
L_{0}=\partial_{x x}+\frac{1}{4}-\frac{3}{2}\left(C_{+}^{* 2}+C_{-}^{* 2}\right),
$$

acting in $L_{\eta}^{2}$. We claim that $L_{0}$ is Fredholm with index 0 for $\eta$ sufficiently small. Since $L_{0}$ is a relatively compact perturbation of the asymptotic operator

$$
L_{0}^{\infty}=\partial_{x x}-\frac{1}{4},
$$

Fredholm properties coincide. In particular, since $L_{0}^{\infty}$ is invertible on $L^{2}, L_{0}$ is Fredholm of index 0 on $L^{2}$. By conjugation with an equivalent smooth weight, one can see that $L_{0}$ on $L_{\eta}^{2}$ is conjugate to an operator $L_{0}^{\eta}$ that smoothly depends on $\eta$ as a closed unbounded operator, so that the Fredholm index 
is constant for small $\eta$; see for instance $[6, \S 2.3]$ for a discussion of Fredholm indices in exponentially weighted spaces.

We next claim that $L_{0}$ has a trivial kernel. Indeed, assuming that $C_{0} \in L_{\eta}^{2}$ belongs to the kernel of $L_{0}$, by taking the $L^{2}$-scalar product

$$
\left\langle L_{0} C_{0}, C_{0}\right\rangle=-\int_{\mathbb{R}}\left(C_{0}^{\prime}(x)\right)^{2} \mathrm{~d} x-\int_{\mathbb{R}}\left(\frac{3}{2}\left(C_{+}^{* 2}+C_{-}^{* 2}\right)-\frac{1}{4}\right)\left(C_{0}(x)\right)^{2} \mathrm{~d} x=0,
$$

we conclude that $C_{0}=0$, since

$$
\frac{3}{2}\left(C_{+}^{* 2}+C_{-}^{* 2}\right)-\frac{1}{4} \geqslant \frac{3}{4}\left(C_{+}^{*}+C_{-}^{*}\right)^{2}-\frac{1}{4} \geqslant 0 .
$$

Consequently, the linear operator $\mathcal{M}_{0}$ is invertible in $X_{\eta}^{0}$.

Finally, the linear operator $\mathcal{M}_{i}$ is also diagonal. Its Fredholm properties, as an operator acting in $X_{\eta}^{i}$, are in fact the same as those of the scalar operator

$$
L_{i}=\partial_{x x}+\frac{1}{4 k_{x}^{2}}-\frac{3}{4 k_{x}^{2}}\left(2 C_{+}^{* 2}+C_{-}^{* 2}\right),
$$

acting in $L_{\eta}^{2}$, that is, without imposing reflection symmetry. In contrast to $L_{0}$, the operator $L_{i}$ is a Fredholm operator with index -1 . We claim that its kernel is trivial. Indeed, if $C_{+} \in L_{\eta}^{2}$ belongs to the kernel of $L_{i}$, then $C_{+}$solves the linear ordinary differential equation

$$
C_{+}^{\prime \prime}+\left(\frac{1}{4 k_{x}^{2}}-\frac{3}{4 k_{x}^{2}}\left(2 C_{+}^{* 2}+C_{-}^{* 2}\right)\right) C_{+}=0 .
$$

In the limit $x=-\infty$, this equation becomes $C_{+}^{\prime \prime}=0$ which implies that solutions of (4.7) do not decay exponentially as $x \rightarrow-\infty$. Consequently, (4.7) does not have solutions in $L_{\eta}^{2}$ and the kernel of $L_{i}$ is trivial. We conclude that $\mathcal{M}_{i}$ is a Fredholm operator with index -1 and trivial kernel. A direct calculation shows that $\left(\mathrm{i}_{+}^{*},-\mathrm{i} C_{-}^{*}\right)$ belongs to the kernel of the $L^{2}$-adjoint of $\mathcal{M}_{i}$, which is one-dimensional, hence spanned by $\left(\mathrm{i} C_{+}^{*},-\mathrm{i} C_{-}^{*}\right)$.

The properties of $\mathcal{M}_{0}, \mathcal{M}_{+}$, and $\mathcal{M}_{-}$above imply the result in the lemma.

\subsection{Persistence of the heteroclinic orbit}

We show that the reversible heteroclinic orbit $\left(0, C_{+}^{*}, C_{-}^{*}\right)$ of $(4.2)$ with $\mu=0$, persists for small $\mu$ as a heteroclinic orbit connecting two periodic orbits $\mathbf{P}_{\mu, \varepsilon}$, as $x \rightarrow \infty$, and $\mathbf{Q}_{\mu, \varepsilon}$, as $x \rightarrow-\infty$. More precisely, we prove the following result.

Theorem 2 For any $\mu$ sufficiently small, there exists $\varepsilon=\varepsilon(\sqrt{\mu}), \varepsilon(0)=0$, such that the system (4.2) possesses a heteroclinic orbit $\mathbf{C}_{\mu}$ connecting the periodic orbit $\mathbf{P}_{\mu, \varepsilon}$, as $x \rightarrow \infty$, to $\mathbf{Q}_{\mu, \varepsilon}$, as $x \rightarrow-\infty$.

Proof. The system (4.2) together with the complex conjugated equations is of the form

$$
\mathcal{F}\left(\mathbf{C}, \overline{\mathbf{C}}, \mu^{1 / 2}\right)=0, \quad \mathbf{C}=\left(C_{0}, C_{+}, C_{-}\right),
$$

and it has the periodic solutions $\mathbf{C}=\mathbf{P}_{\mu, \varepsilon}$ and $\mathbf{C}=\mathbf{Q}_{\mu, \varepsilon}$ for $\mu$ and $\varepsilon$ sufficiently small, and the heteroclinic solution $\mathbf{C}=\left(0, C_{+}^{*}, C_{-}^{*}\right)$ for $\mu=0$. 
We look for solutions of (4.8) of the form

$$
\mathbf{C}(x)=\mathrm{e}^{\mathrm{i} \varepsilon x} \mathbf{C}^{*}(x)+\chi(x) \widetilde{\mathbf{P}}_{\mu, \varepsilon}(x)+\left(\mathcal{R}\left(\chi \widetilde{\mathbf{P}}_{\mu, \varepsilon}\right)\right)(-x)+\mathbf{V}(x),
$$

in which

$$
\mathbf{C}^{*}=\left(0, C_{+}^{*}, C_{-}^{*}\right), \quad \widetilde{\mathbf{P}}_{\mu, \varepsilon}=\mathbf{P}_{\mu, \varepsilon}-\left(0, \frac{1}{\sqrt{3}}, 0\right) \mathrm{e}^{\mathrm{i} \varepsilon x},
$$

$\chi: \mathbb{R} \rightarrow[0,1]$ is a smooth function such that

$$
\chi(x)=1, \text { if } x \geqslant M, \quad \chi(x)=0, \text { if } x \leqslant m,
$$

for some positive constants $m<M$, and $(\mathbf{V}, \overline{\mathbf{V}})$ belongs to the space $\mathcal{Y}_{\eta}^{r}=\mathcal{Y}_{\eta} \cap \mathcal{X}_{\eta}^{r}$. Substituting (4.9) into (4.8) leads to an equation of the form

$$
\mathcal{T}\left(\mathbf{V}, \overline{\mathbf{V}}, \varepsilon, \mu^{1 / 2}\right)=0
$$

We construct a solution $(\mathbf{V}(\mu), \varepsilon(\mu))$ of this equation, for sufficiently small $\mu$, using the implicit function theorem.

We claim that $\mathcal{T}\left(\mathbf{V}, \overline{\mathbf{V}}, \varepsilon, \mu^{1 / 2}\right) \in \mathcal{X}_{\eta}^{r}$ for $(\mathbf{V}, \overline{\mathbf{V}}) \in \mathcal{Y}_{\eta}^{r}$. Indeed, as $x \rightarrow \infty$ (resp. $\left.x \rightarrow-\infty\right)$ the difference $\mathbf{C}-\mathbf{P}_{\mu, \varepsilon}$ (resp. $\mathbf{C}-\mathbf{Q}_{\mu, \varepsilon}$ ) decays exponentially to 0 , with the same decay rate as $\mathbf{V}$. Since $\mathcal{F}\left(\mathbf{P}_{\mu, \varepsilon}, \overline{\mathbf{P}}_{\mu, \varepsilon}, \mu^{1 / 2}\right)=\mathcal{F}\left(\mathbf{Q}_{\mu, \varepsilon}, \overline{\mathbf{Q}}_{\mu, \varepsilon}, \mu^{1 / 2}\right)=0$ this implies that $\mathcal{T}\left(\mathbf{V}, \overline{\mathbf{V}}, \varepsilon, \mu^{1 / 2}\right) \in \mathcal{X}_{\eta}$. Furthermore, $(\mathbf{C}, \overline{\mathbf{C}})$ is reversible, if $(\mathbf{V}, \overline{\mathbf{V}})$ is reversible, so that $\mathcal{T}\left(\mathbf{V}, \overline{\mathbf{V}}, \varepsilon, \mu^{1 / 2}\right) \in \mathcal{X}_{\eta}^{r}$.

Next, notice that

$$
\mathcal{T}(0,0,0,0)=\mathcal{F}\left(\mathbf{C}^{*}, \overline{\mathbf{C}^{*}}, 0\right)=0, \quad D_{\mathbf{V}} \mathcal{T}(0,0,0,0)=\mathcal{L}_{*}
$$

and

$$
D_{\varepsilon} \mathcal{T}(0,0,0,0)=\mathcal{L}_{*}\left(\begin{array}{c}
i x \mathbf{C}^{*} \\
-i x \mathbf{C}^{*}
\end{array}\right)=\left(\begin{array}{c}
2 i \mathbf{C}^{* \prime} \\
-2 i \mathbf{C}^{* \prime}
\end{array}\right)
$$

Recall that the kernel of the $L^{2}$-adjoint of $\mathcal{L}^{*}$ is spanned by $\left(0, \mathrm{i} C_{+}^{*},-\mathrm{i} C_{-}^{*}, 0,-\mathrm{i} C_{+}^{*}, \mathrm{i} C_{-}^{*}\right)$, and notice that the $L^{2}$-scalar product of this vector with $\left(2 i \mathbf{C}^{* \prime},-2 i \mathbf{C}^{* \prime}\right)$ is given by

$$
2 \int_{\mathbb{R}}\left(2 C_{+}^{* \prime}(x) C_{+}^{*}(x)-2 C_{-}^{* \prime}(x) C_{-}^{*}(x)\right) \mathrm{d} x=2 \int_{\mathbb{R}}\left(C_{+}^{* 2}(x)-C_{-}^{* 2}(x)\right)^{\prime} \mathrm{d} x=-\frac{4}{\sqrt{3}} \neq 0 .
$$

This shows that $\left(2 i \mathbf{C}^{* \prime},-2 i \mathbf{C}^{* \prime}\right)$ does not belong to the range of $\mathcal{L}^{*}$, which together with the fact that $\mathcal{L}_{*}$ is an injective Fredholm operator with index -1 implies that the differential $D_{(\mathbf{V}, \varepsilon)} \mathcal{T}(0,0,0,0)$ is bijective. The result in the lemma now follows from the implicit function theorem.

Remark 4.2 A closer inspection shows that the key component of the persistence proof is the computation of the Melnikov integral (4.10). Transversality and persistence follow from the fact that this Melnikov integral does not vanish. On the other hand, the direction generating transversality in this argument is the centre direction, more precisely the variation of the wavenumber in the background. From this point of view, robustness of the grain boundary is equivalent to (transverse) wavenumber selection. 
The result in Theorem 1 is an immediate consequence of Theorem 2. Indeed, recall that the choice $k_{*} \in(1 / 2,1)$ corresponds to angles $\alpha \in(\pi / 3, \pi)$. Since $\varepsilon=\mathrm{O}(\sqrt{\mu})$, we find that the angle of the selected grain boundary is $\alpha+\mathrm{O}(\mu)$, and that the wavenumber of the asymptotic rolls is $k_{x}+\mathrm{O}(\mu)$. In particular, we can invert the dependence of the angle $\alpha$ and the parameter $k_{*}$ and find $k_{*}$ and $k_{x}$ as smooth functions of the angle $\alpha$, which is the desired wavenumber selection mechanism. This gives to leading order in $\mu$ the constant wavenumber $\sqrt{k_{x}^{2}+k_{y}^{2}}=1+\mathrm{O}(\mu)$.

Remark 4.3 Since rolls exist within a band of width $\mathrm{O}(\sqrt{\mu})$, the asymptotic wavenumber $\sqrt{k_{x}^{2}+k_{y}^{2}}=$ $1+\mathrm{O}(\mu)$ is in the centre of the existence band to leading order. It would be interesting to compute the leading-order term in this expansion and compare with the zigzag instability boundary, which is equally located on a curve with wavenumber $1+\mathrm{O}(\mu)$.

\section{Discussion}

We showed existence of grain boundaries for not-too-small angles $\alpha>\pi / 3$ between rolls. We also showed that grain boundaries select wavenumbers. More precisely, for any given angle, there exists a grain boundary between rolls with a specific wavenumber that depends on the angle (and explicit system parameters such as $\mu$ ). In the following, we discuss some generalizations and two major open questions relating to stability and bifurcations of grain boundaries.

Beyond Swift-Hohenberg. We mentioned in the introduction that we expect our results to generalize to pattern-forming equations such as reaction-diffusion systems or Rayleigh-Bénard convection. In fact, the linear analysis and the reduction procedure would apply only assuming an isotropic system with marginally stable modes $\mathrm{e}^{\mathrm{i}(\underline{k} \cdot \underline{x})+\lambda(|\underline{k}|) t)}$, and $\lambda(\kappa) \sim \mu-\kappa^{2}$. The reduced equations will generally be of the type (4.2), even when quadratic terms are present, since the assumption on the angle effectively excludes the three-mode interaction that generates hexagons. In fact, hexagons require $\alpha=\pi / 3$. Quadratic terms do however change the cubic coefficients in (4.2). In fact, those coefficients do in general depend on the angle $\varphi_{-}-\varphi_{+}$. We expect a variety of intriguing phenomena in such situations, including bifurcations towards grain boundaries that involve more than two modes at leading order.

Wavenumber selection - beyond small amplitude. The statement of our main result, Theorem 1, emphasizes the wavenumber selection aspect of the grain boundaries that we found. Such wavenumber selection mechanisms have been observed experimentally and discussed at the level of amplitude equations; see [13] and the references therein. Our analysis relates this wavenumber selection mechanism to a geometric transversality criterion for a heteroclinic solution. A similar connection has been noticed in $[23,18]$ : in oscillatory media, localized structures that emit wave trains typically select wavenumbers in the far-field. In fact, such sources of wave trains can be viewed as heteroclinic orbits in a spatial dynamics description, and transversality of such heteroclinic orbits implies wavenumber selection in the far field. A key difference between such sources and the general type of problem considered here is that the dispersion relation of the patterns in the far-field is trivial, that is, group velocities vanish for all wavenumbers. One could however consider symmetric grain boundaries more generally, far from onset for not necessarily small $\mu$. Without showing existence, one can then predict Fredholm 
properties of the linearization at such a grain boundary solution following the counting arguments in [19]. Stability of the asymptotic patterns alone then guarantees that the linearization is Fredholm of index -1 , as shown in our particular case in Lemma 4.1. If one assumes that the kernel is trivial as in our case, robustness of such grain boundaries follows if the Melnikov-type derivative with respect to the asymptotic wavenumber does not belong to the range of the linearization. In this sense, wavenumber selection for grain boundaries is a typical property, even far from onset.

Stability. We did not attempt to perform a stability analysis. The fact that heteroclinic orbits are constructed in [22] as minimizers of the energy associated with the reduced system (4.6) provides only a weak indicator for stability. In fact, the corresponding action functional is only minimized for real amplitudes. Also, the description considers periodic perturbations in $y$ only. On an even more elementary level, it is not clear that the rolls that are selected by the grain boundary are stable. As we pointed out in Remark 4.3, the wavenumber selected by grain boundaries is to leading order identical to the critical wavenumber $k=1$, thus coinciding to leading order with the zigzag instability boundary. Of course, in the leading-order amplitude description, both curves actually coincide and one is led to associate perfect wavenumber selection with grain boundaries [13]. We are, however, unable to see a reason why this perfect selection would happen at higher order. Deviations of the selected wavenumber from the zigzag-critical wavenumber could possibly lead to quite intriguing dynamics. One could even envision that stability of the selected rolls depends on the angle of the grain boundaries, thus generating complex dynamics of zigzag patterns. The results in [8] show that the selected wavenumber corresponds to stable roll solutions, that is, it is larger than the zigzag critical wavenumber, for large angles $\alpha \lesssim \pi$

- in particular showing that grain boundaries do not select the "perfect" wavenumber at higher order for large angles.

Bifurcations. Interesting bifurcations of grain boundaries have been analyzed in [3, 4] and [5]. The analysis there is based on the phase-diffusion equation [2], treating amplitude defects as singularities. Intriguing results are found for smaller angles, where grain boundaries change type, effectively resembling dislocations, or pairs of convex and concave disclinations. While the results there are derived for large amplitudes, far from onset, and should be expected to hold with some universality, it is an interesting problem to analyze such transitions in the framework presented here.

A related question concerns the restriction to non-small angles, $\alpha>\pi / 3$. For smaller values of $\alpha$, resonant wavenumbers enlarge the dimension of the reduced system. Similarly, asymmetric grain boundaries typically involve more modes. We intend to analyze such situations in future work.

\section{References}

[1] M. Cross and P. Hohenberg. Pattern Formation Out of Equilibrium. Rev. Modern Phys. 65 (1993), $851-1112$.

[2] M. Cross and A. Newell. Convection Patterns in Large Aspect Ratio Systems. Physica D 10 (1984), 299-328. 
[3] N. Ercolani, R. Indik, A. Newell, and T. Passot. The geometry of the phase diffusion equation. J. Nonlinear Sci. 10 (2000), 223-274.

[4] N. Ercolani, R. Indik, A. Newell, and T. Passot. Global description of patterns far from onset: a case study. Complexity and nonlinearity in physical systems (Tucson, AZ, 2001). Phys. D 184 (2003), 127-140.

[5] N. Ercolani and S. Venkataramani. A variational theory for point defects in patterns. J. Nonlinear Sci. 19 (2009), 267-300.

[6] B. Fiedler and A. Scheel. Spatio-Temporal Dynamics of Reaction-Diffusion Patterns. In Trends in Nonlinear Analysis, M. Kirkilionis, S. Krmker, R. Rannacher, F. Tomi (Eds.), Springer-Verlag, Berlin, 2003.

[7] M. Haragus and G. Iooss. Local bifurcations, center manifolds, and normal forms in infinite dimensional dynamical systems. Universitext. Springer-Verlag London, Ltd., London; EDP Sciences, Les Ulis; 2011.

[8] M. Haragus and A. Scheel. Interfaces between rolls in the Swift-Hohenberg equation. Int. J. Dyn. Syst. Differ. Equ. 1 (2007), 89-97.

[9] M. Haragus and A. Scheel. Dislocations in an anisotropic Swift-Hohenberg equation. To appear in Comm. Math. Phys.

[10] G. Iooss and M. Adelmeyer. Topics in bifurcation theory and applications. Second edition. Advanced Series in Nonlinear Dynamics, 3. World Scientific Publishing Co., Inc., River Edge, NJ, 1998.

[11] G. James and Y. Sire. Center manifold theory in the context of infinite one-dimensional lattices. The Fermi-Pasta-Ulam problem, 208-238, Lecture Notes in Phys. 728, Springer, Berlin, 2008.

[12] K. Kirchgässner. Wave-solutions of reversible systems and applications. J. Differential Equations 45 (1982), no. 1, 113-127.

[13] B. Malomed, A. Nepomnyashchy, and M. Tribelsky. Domain boundaries in convection patterns. Phys. Rev. A 42 (1990), 7244-7263.

[14] A. Mielke. Instability and stability of rolls in the Swift-Hohenberg equation. Comm. Math. Phys. 189 (1997), 829-853.

[15] A. Mielke. Hamiltonian and Lagrangian flows on center manifolds. With applications to elliptic variational problems. Lecture Notes in Mathematics 1489. Springer-Verlag, Berlin, 1991.

[16] A. Mielke. The Ginzburg-Landau equation in its role as a modulation equation. Handbook of dynamical systems, Vol. 2, 759-834, North-Holland, Amsterdam, 2002.

[17] A. Newell. The dynamics of patterns: a survey. Propagation in systems far from equilibrium (Les Houches, 1987), 122-155, Springer Ser. Synergetics, 41, Springer, Berlin, 1988. 
[18] B. Sandstede and A. Scheel. Defects in oscillatory media: toward a classification. SIAM J. Appl. Dyn. Syst. 3 (2004), 1-68.

[19] B. Sandstede and A. Scheel. Relative Morse indices, Fredholm indices, and group velocities. Discr. Cont. Dyn. Sys. 20 (2008), 139-158.

[20] A. Scheel. Radially symmetric patterns of reaction-diffusion systems. Mem. Amer. Math. Soc. 165 (2003).

[21] G. Schneider. Validity and limitation of the Newell-Whitehead equation. Math. Nachr. 176 (1995), 249-263.

[22] G. van den Berg and R. van der Vorst. A domain-wall between single-mode and bimodal states. Diff. Int. Equations 13 (2000), 369-400.

[23] W. van Saarloos and P. Hohenberg. Fronts, pulses, sources and sinks in generalized complex GinzburgLandau equations. Physica D 56 (1992), 303-367.

[24] A. Vanderbauwhede and G. Iooss. Center manifold theory in infinite dimensions. Dynamics reported: expositions in dynamical systems, 125-163, Dynam. Report. Expositions Dynam. Systems (N.S.) 1, Springer, Berlin, 1992.

[25] A. Vanderbauwhede. Centre manifolds, normal forms and elementary bifurcations. Dynamics reported, Vol. 2, 89-169, Dynam. Report. Ser. Dynam. Systems Appl. 2, Wiley, Chichester, 1989. 